\title{
Diversidad y taxonomía de roedores de Nicaragua
}

\section{Diversity and taxonomy of rodents in Nicaragua}

\section{Javier Monge}

Centro de Investigaciones en Protección de Cultivos (CIPROC), Escuela de Agronomía, Universidad de Costa Rica. javier.monge@ucr.ac.cr

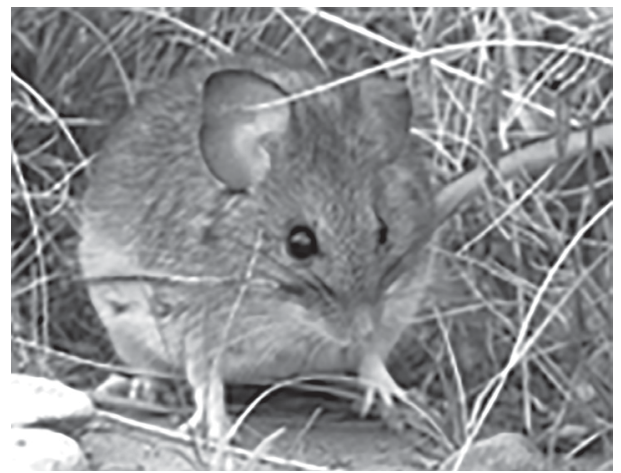

\section{RESUMEN}

Los roedores constituyen el grupo más diverso de los mamíferos a nivel mundial. Se dispone de suficiente información de muchas de las especies de este grupo, sin embargo, a nivel local puede carecerse de información mínima de algunas especies. En el caso de Nicaragua, se dispone de relativamente poca información documentada o de fácil acceso sobre el tema. Por medio de una revisión bibliográfica de referencias mundiales y regionales se elaboró una lista de especies de roedores presentes en Nicaragua. De cada una de las especies de roedores presentes en Nicaragua se recabó información básica, para su reconocimiento, así como su relación con las personas. En Nicaragua actualmente se reportan 39 especies de roedores, que incluyen ratas y ratones, ardillas, taltuza, puercoespín, guatusa y tepezcuintle, de las cuales dos especies son endémicas para Nicaragua. Se considera que la información incluida en este documento ofrece un panorama general acerca de la taxonomía y diversidad de este grupo en Nicaragua. A su vez, se espera que en la medida que los profesionales y técnicos relacionados con este grupo taxonómico dispongan de más información, se propicie el desarrollo de estudios locales que amplíen el grado de conocimiento de este grupo. La disponibilidad de información básica local es fundamental para la toma de decisiones de manejo, ya sea para fines de control de algunas especies o de protección de otras.

Palabras clave: rodentia, Nicaragua, taxonomía, roedores plaga, especies endémicas.

Recibido: 20 de enero 2015

Aceptado: 22 de abril 2016

\begin{abstract}
Rodents are the most diverse group of mammals worldwide. Of this group, there is enough information for many species, however, at the local level, information is scarce for some species. In the case of Nicaragua, there is relatively little documented information or easily accessible on the subject. A list of species of rodents present in Nicaragua was developed through a literature review of global and regional references. Each of the species of rodents present in Nicaragua collected basic information, for its recognition, as well as its relationship with the people. For Nicaragua are currently reported 39 species of rodents, including rats and mice, squirrels, pocket gopher, porcupine, agouti and paca, of which two species are endemic to Nicaragua. It is considered that the information contained in this document provides a general overview about the taxonomy and diversity of this group in Nicaragua. To its time, is expected that in the measure that the professional and technical related with this group taxonomic have of more information, is conducive to the development of studies local that expand the grade of knowledge of this group. The availability of local basic information is essential for making management decisions, either for control of some species or protection of others.
\end{abstract}

Keywords: Rodentia, Nicaragua, taxonomy, rodent pest, endemic species 
$\mathrm{L}$

os roedores constituyen el grupo más abundante de los mamíferos a nivel mundial, con una representación del $42,55 \%$. La cantidad de mamíferos es de 6017 especies de los cuales 2560 son roedores (Wilson y Reeder, 2005; Reeder et al., 2007), cuya tendencia es hacia el crecimiento, dados los descubrimientos de nuevas especies.

En la actualidad, el conocimiento de este grupo taxonómico es amplio, sin embargo, para el público en general es limitado, lo cual también ocurre con profesionales no especialistas en el tema, pero que en alguna medida relacionado con el mismo. Como en cualquier otro campo del conocimiento, la carencia de información propicia a que se hagan generalizaciones que pueden estar alejadas de la realidad. Así, por ejemplo, cuando se hace referencia a los roedores, muchas veces se asocia solo con ratas y ratones, y dentro de éstos, a aquellos que ocasionan perjuicios a los intereses de las personas. Por lo tanto, el término roedor se relaciona con especies dañinas, lo cual evidentemente representa un concepto erróneo basado en un conocimiento limitado de este grupo.

La información documentada sobre el tema de roedores en Nicaragua es escasa o poco accesible, lo cual conlleva a referido conocimiento limitado sobre el tema, lo que a su vez, retrasa el avance en este campo. En la medida en que se desconozca la diversidad de especies en una región o país, así como la relación de las especies con su medio ambiente $\mathrm{y}$ en particular con el ser humano, propicia a que se tomen acciones no apropiadas o bien que no se actúe cuando la situación lo amerite. Una generalización de los perjuicios que ocasiona alguna especie de roedor, como si cualquier otra especie también lo provoca por el hecho de ser roedor, puede afectar a poblaciones con poblaciones reducidas o incluso a especies endémicas.

El propósito de este documento es el de dar a conocer la diversidad y taxonomía de los roedores en Nicaragua, así como ofrecer un conocimiento básico de la ecología de las especies, sin pretender dar el tema por agotado. En algunos casos se hace referencia a la necesidad de realizar estudios sobre algunas especies, dada la carencia de información documentada que sustente la toma de decisiones o bien que sirva de base para estudios más detallados. Es evidente que algunos estudios deben hacerse en Nicaragua cuando se trate de especies endémicas, pero también se justifica realizar estudios dentro del país, ya que si bien son especies cuya distribución geográfica supere los límites del país, conviene disponer infor- mación local para la toma de decisiones que la situación particular lo requiera.

\section{METODOLOGÍA}

Se recabó información sobre la diversidad de roedores de Nicaragua, basada en documentos de referencia sobre mamíferos a nivel mundial (Wilson y Reeder, 2005; Reeder et al., 2007), así como a nivel regional para Mesoamérica (Reid, 2009).

La lista de especies de roedores ha sido organizada por familias, para su ubicación taxonómica a este nivel; sin embargo, a nivel de género o especie se han presentado modificaciones a través del tiempo, y es posible que algunas de las especies aún sea reclasificadas en la medida que nuevos criterios y técnicas permitan una clasificación más precisa. De cada de las especies se aporta información para su reconocimiento preliminar y además se incluye información sobre el tipo de hábitat que ocupa, y en algunos casos, su ubicación dentro del territorio de Nicaragua.

Adicionalmente, se incluye información sobre aspectos ecológicos y su relación con el ser humano, ya sea como especie dañina, de consumo o bien por su carácter endémico que requiera un cuidado especial para su preservación.

\section{RESULTADOS Y DISCUSIÓN}

En Mesoamérica (Sureste de México y América Central), se registra un total de 362 especies de mamíferos, de las cuales 31 son especies marinas (Cuadro 1). A diferencia a lo que ocurre a nivel mundial, en Mesoamérica el grupo más representado es el de los murciélagos (Orden Chiroptera) con un total de 144 especies $(39,78 \%)$. El siguiente grupo en abundancia de especies es el de los roedores (Orden Rodentia) con 105 especies $(29,01 \%)$, siendo la mayoría ratas y ratones, aunque también se incluye ardillas, taltuzas, el tepezcuintle, las guatusas, puercoespines y el capibara, siendo este último el roedor más grande del mundo (Reid, 2009).
Cuadro 1. Órdenes de mamíferos de Mesoamérica

\begin{tabular}{lrrr}
\hline Orden & Géneros & Especies & Especies \% \\
\hline Chiroptera (Murciélagos) & 67 & 144 & 39.7 \\
Rodentia (Roedores) & 42 & 105 & 29.0 \\
Cetacea (Ballenas, delfines, cachalotes) & 19 & 31 & 8.5 \\
Carnivora (Coyote, felinos, mapache y otros) & 19 & 26 & 7.1 \\
Soricomorpha (Musarañas) & 2 & 15 & 4.1 \\
Didelphimorphia (Zarigüeyas, zorros) & 10 & 14 & 3.8 \\
Primates (Monos) & 6 & 8 & 2.2 \\
Artiodactyla (Saínos, venados) & 4 & 6 & 1.6 \\
Pilosa (Osos hormigueros, perezosos) & 5 & 5 & 1.3 \\
Lagomorpha (Liebres y conejos) & 2 & 4 & 1.1 \\
Cingulata (Armadillos) & 2 & 2 & 0.5 \\
Sirenia (Manatí) & 1 & 1 & 0.2 \\
Perissodactyla (Danta) & 1 & 1 & 0.2 \\
& & & \\
\hline
\end{tabular}


En Nicaragua, el Orden Rodentia está constituido por 9 familias, 26 géneros y 39 especies. De estas, tres especies son ardillas, una taltuza, dos ratones semiespinosos, 25 especies de ratas y ratones de campo, un puercoespín, una guatusa, un tepezcuintle y dos ratas espinosas. Además están presentes tres especies, de una familia exótica (Muridae) de distribución cosmopolita (cuadro 2).

Cuadro 2. Especies de roedores de Nicaragua

\begin{tabular}{|c|c|c|}
\hline SCIURIDAE (Ardillas) & CRICETIDAE $^{1}$ & MURIDAE $^{2}$ \\
\hline Sciurus variegatoides & Oryzomys couesi & Rattus rattus \\
\hline S. deppei & O. dimidiatus (endémica) & R. norvegicus \\
\hline S. richmondi (endémica) & $\begin{array}{l}\text { Transandinomys bolivaris } \\
\text { Handleyomys rostratus }\end{array}$ & Mus musculus \\
\hline GEOMYIDAE (Taltuzas) & H. alfaroi & ERETHIZONTIDAE (Puercoespines \\
\hline $\begin{array}{l}\text { Orthogeomys matagalpae } \\
\text { (Heterogeomys cherriei) }\end{array}$ & H. saturatior & Sphiggurus mexicanus \\
\hline $\begin{array}{l}\text { HETEROMYIDAE } \\
\text { Liomys salvini } \\
\text { Heteromys desmarestianus }\end{array}$ & $\begin{array}{l}\text { Sigmodontomys alfari } \\
\text { Melanomys caliginosus } \\
\text { Oligorizomys fulvecens } \\
\text { Sigmodon hirsutus } \\
\text { Tylomys nudicaudus } \\
\text { Ototylomys phyllotis } \\
\text { Nyctomys sumichrasti } \\
\text { Neotoma chrysomelas } \\
\text { Baiomys musculus } \\
\text { Scotinomys teguina } \\
\text { Reithrodontomys sumichrasti } \\
\text { R. fulcescens } \\
\text { R. gracilis } \\
\text { R. mexicanus } \\
\text { R. brevirostris } \\
\text { R. paradoxus } \\
\text { Peromyscus mexicanus } \\
\text { P. gymnotis } \\
\text { P. stirtoni }\end{array}$ & $\begin{array}{l}\text { DASYPROCTIDAE (Guatusas) } \\
\text { Dasyprocta punctata } \\
\text { CUNICULIDAE (Tepezcuintle) } \\
\text { Cuniculus paca } \\
\text { ECHIMYIDAE (Ratas espinosas) } \\
\text { Proechimys semispinosus } \\
\text { Haplomys gymnurus }\end{array}$ \\
\hline
\end{tabular}

${ }^{1}$ Ratas y ratones del nuevo mundo, ${ }^{2}$ Ratas y ratones exóticos, ${ }^{3}$ Ratones semiespinosos.

En Nicaragua se encuentran cinco subespecies $(S . v$. belti, S. v. underwoodi, S. v. dorsalis, S. v. adolphei y $S$. v. boothiae) (Hall, 1981), teniendo las tres primeas mayor distribución. S. v. belti es de color café con vientre anaranjado y se encuentra en el centro hacia el oeste del país, incluyendo los departamentos de Zelaya, y la parte oeste de los departamentos Río San Juan, Chontales, Boaco, Matagalpa y Jinotega. S. v. underwoodi tiene tonalidades de gris oscuro con el vientre blanco y se encuentra de la parte central del país hacia la costa pacífica, mientras que S. v. dorsalis es de color blanco en los lados con el dorso negro y se encuentra en la parte noreste desde el Lago de Nicaragua, en los departamentos de Masaya, Managua, Jinotepe y León. Habita en bosques, así como en plantaciones y alrededores de áreas agrícolas. Se le considera plaga para diferentes especies forestales y cultivos agrícolas (Monge, 2009).

$S$. deppei es más pequeña que $S$. variegatoides, su cuerpo mide entre 18 y $22 \mathrm{~cm}$, y la cola entre 15 y $20 \mathrm{~cm}$, con un peso entre $190 \mathrm{y}$ 220 g. Su coloración es café con el vientre blanco grisáceo.Se le encuentra

De estas 39 especies, resalta la ardilla Sciurus richmondi y la rata arrocera nicaragüense Oryzomys dimidiatus, las cuales son endémicas para Nicaragua.

A continuación se hace una breve referencia a los roedores presentes en Nicaragua, incluyendo aspectos para su reconocimiento, hábitat que ocupan, así como anotaciones de la relación de esta fauna con los intereses del ser humano.

Ardillas. En Nicaragua existen tres especies de ardillas, todas del género Sciurus (S. variagatoides, S. deppei y S. richmondi).

S. variegatoides es la de mayor tamaño y distribución, encontrándose en todo el territorio. Su cuerpo mide entre 24 y $30 \mathrm{~cm}$, y su larga cola es de un tamaño similar. Pesa entre 500 y 900 g. Esta especie tiene 15 subespecies, cuya coloración varía considerablemente entre éstas, lo que puede llevar a la confusión al considerárseles diferentes especies. en la parte central del país, desde el norte hasta el sur. Habita en bosques siempreverdes y bosques deciduos. Puede ingresar a áreas agrícolas y convertirse en plaga del maíz y otros cultivos (Best, 1995; Reid, 2009). S. richmondi es de un tamaño similar a $S$. deppei, con un largo de cuerpo de 16 a 22 $\mathrm{cm}$, largo de cola de $13-18 \mathrm{~cm}$ y el peso oscila entre $235 \mathrm{y}$ 270 g. Su coloración es café y vientre anaranjado. Se encuentra en un área reducida en la vertiente atlántica, en bosques siempreverdes, en bosques al borde de ríos y en plantaciones. Es una especie endémica de Nicaragua y sus poblaciones son reducidas, por lo que requiere protección. Se dispone de poca información de esta especie (Jones y Genoways, 1975), por lo que es conveniente realizar estudios de su biología y ecología.

Taltuzas. Hasta hace poco se consideraba que la taltuza Orthogeomys matagalpae era la única que se encuentra en 
Nicaragua, y solo se distribuye en unos pequeños sectores de Nicaragua y Honduras. Es de color café o marrón oscuro con una mancha blanca en la cabeza. Su cuerpo mide entre 16 y $24 \mathrm{~cm}$, y la cola es corta y desnuda y mide entre 7 y 12 $\mathrm{cm}$. Pesa alrededor de $290 \mathrm{~g}$. Sin embargo, recientemente se propuso una reclasificación taxonómica que recupera el subgénero Heterogeomys, y además se considera sinonimia de Ortogeomys matagalpae con lo que fue O. cherriei, quedando ahora solo (Spradling et al., 2016). En Nicaragua, se reporta que daña raíces de café (Straver, 1996) aunque es posible que dañe otros cultivos agrícolas, tal como se reporta para Costa Rica (Monge, 2009), ahora que se consideran la misma especie llamada $H$. cherriei. Se dispone de poca información de esta especie en Nicaragua, por lo que es conveniente realizar estudios de la misma.

Ratones heterómidos (semiespinosos). En Nicaragua se registran dos especies de la familia Heteromyidae, cuyos miembros se conocen como ratones semiespinosos. Los miembros de esta familia se caracterizan por tener bolsas en las mejillas, al igual que las taltuzas (Geomyidae), que usan para transportar semillas y otros alimentos, así como materiales de construcción para sus nidos (Fleming, 1991a).

Liomys salvini es un ratón pequeño con largo de cuerpo entre 10 y $14 \mathrm{~cm}$, con largo de cola similar a la del cuerpo, y pesa entre 30 y $65 \mathrm{~g}$. Su pelaje es áspero como suaves espinas en el dorso de color grisáceo, y el vientre es de color gris claro. Habita en bosques deciduos y campos enmalezados.

Heteromys desmarestianus es un ratón mediano, con largo de cuerpo entre 12 y $15 \mathrm{~cm}$, y la cola entre 13 y 20 $\mathrm{cm}$, con un peso entre 46 y $87 \mathrm{~cm}$. Presenta un dimorfismo sexual, pesando los machos un tercio más que las hembras (Fleming, 1991b). El pelaje es similar a espinas suaves de color café negrusco y pelos de color ocre, con vientre blanco amarillento. Habita en bosques siempreverdes, semideciduos $\mathrm{y}$ de crecimiento secundario.

Ratas y ratones cricétidos. Incluye 25 especies de ratas y ratones nativos pertenecientes a la familia Cricetidae. A continuación se hace referencia a los géneros de esta familia presentes en Nicaragua. La información que se presenta es de carácter general, por lo que su precisión es limitada, principalmente en lo referente a las indicaciones de la coloración de las especies que puede variar entre los individuos a través del área de distribución de la especie, que en algunos casos puede ser muy amplia. De igual manera, las medidas pueden incluir datos provenientes de individuos juveniles y adultos o solo adultos, lo cual depende de las fuentes de información.

Oryzomys. Este género incluye algunas de las llamadas ratas arroceras. En Nicaragua se encuentran dos especies $O$. couesi y $O$. dimidiatus.

O. couesi es un ratón mediano de color café a café anaranjado con la parte ventral blancuzca. Su cuerpo mide de $10-15 \mathrm{~cm}$, la cola de $11-15 \mathrm{~cm}$ y pesa entre 43 y $82 \mathrm{~g}$. Habita en hábitats variados en todo el territorio y puede invadir y atacar cultivos de arroz y caña de azúcar (Sánchez y Martínez 2000).

O. dimidiatus es de color café grisáceo con negro $\mathrm{y}$ en la parte ventral gris plateado. El cuerpo mide de 12-13 $\mathrm{cm}$, la cola de 11-15 y pesa $46 \mathrm{~g}$. Es una especie endémica de Nicaragua que habita en un pequeño sector de la zona atlántica. Se dispone de muy poca información de esta especie, por lo que conviene realizar estudios de su biología y ecología.

Transandinomys. Este género está representado por la especie $T$. bolivaris, previamente clasificado como Oryzomys talamancae (Weksler et al., 2006), aunque Reid (2009) indica que antes era conocido como Oryzomys bombycinus. Su pelaje es de color café oscuro a café amarillento y la parte ventral blanco grisáceo. Mide entre 10 y $14 \mathrm{~cm}$, la cola de 10-13, con peso de 39-75 g. Habita en bosques maduros y siempre verdes en la costa atlántica.

Handleyomys. Género representado por tres especies $(H$. rostratus, $H$. alfaroi y $H$. saturatior) que al igual que los dos géneros anteriores, a sus miembros se les conoce como ratas arroceras. H. rostratus es de color café rojizo anaranjado y mide $9-12 \mathrm{~cm}$, la cola de $10-14 \mathrm{~cm}$ y pesa de 29-58 g. Habita en orillas de bosques, cafetales arbolados y en plantaciones de caña de azúcar en la zona del Pacífico de Nicaragua.

$H$. alfaroi es de color café amarillento a café rojizo oscuro, con la parte ventral blanco grisáceo, mide de 8,5$11,5 \mathrm{~cm}$, la cola de 9-12 cm, y pesa entre 22-44 g. Habita en bosques siempre verdes, semideciduos y orillas de bosques.

H. saturatior es de color café oscuro a negruzco y el vientre blanco grisáceo. Mide de 9-10 cm, la cola de 10-12 $\mathrm{cm}$, con un peso de 21-32 g. Habita en bosques nubosos y bosques de pinos.

Sigmodontomys. Representado en Nicaragua por la especie $S$. alfaroi, la cual es de color café rojizo oscuro y la parte ventral café claro o blancuzco. Mide de $12-16 \mathrm{~cm}$, la cola de 14,5-18,5 cm y pesa entre 63 y $111 \mathrm{~g}$. Habita en bosques secundarios, cultivos de caña de azúcar y pantanos.

Melanomys Representado por la especie M. caliginosus, de color café oscuro casi negro y la parte ventral café anaranjado. Mide de 11-13,5 cm, la cola de 8,5 $-11 \mathrm{~cm}$, pesa 40-74 g. Habita en charrales y bosques secundarios y orillas de bosque. También se ha encontrado en pastizales (Wingching-Jones et al., 2009).

Oligorizomys Representado por la especie $O$. fulvecens. Es un ratón pequeño de color anaranjado grisáceo con negro y el vientre blanco con café claro. Mide de 6-10 cm y su cola de 8-12,5 cm, con un peso de 11-16 g. Habita en matorrales y orillas de bosques.

Sigmodon. En Nicaragua esta especie está representado por la especie $S$. hirsutus, anteriormente considerada como subespecie de Sigmodon hispidus. Conocida como rata cañera o rata de campo. Su pelaje es de color café grisáceo con pelos café amarillento, con la parte ventral blanco grisá- 
ceo. El cuerpo mide de 14-16,5 cm, la cola de 9-13 cm, con peso entre 50 y 200 g. Habita en bosques abiertos de crecimientos secundario, pastizales, áreas agrícolas y manglares. Es la principal plaga vertebrada agrícola atacando especies forestales y una gran variedad de cultivos agrícolas.

Tylomys. Género representado por T. nudicaudus. Es una rata grande, de tamaño similar a la rata gris o de caño (Rattus norvegicus). Su coloración es gris o café grisáceo y la parte ventral blanco amarillento. Su cuerpo mide 19-26 $\mathrm{cm}$, con un tamaño de cola similar y pesa $325 \mathrm{~g}$. Habita en bosques maduros siempreverdes y bosques semideciduos.

Ototylomys Representado solo por la especie $O$. phyllotis. Su pelaje es café grisáceo con tonalidades oscuras a claras, con la parte ventral blanco amarillento. Mide de 12,5$16,5 \mathrm{~cm}$, la cola de $11-17,5$ y pesa de $34-89 \mathrm{~g}$. Habita en una gran variedad de ambientes desde bosques deciduos y secundarios de zonas secas hasta bosques de tierras altas húmedos.

Nyctomys Género representado por la especie $N$. sumichrasti. Es de color anaranjado a café amarillento con la parte ventral blanco amarillento. Su cuerpo mide de 10-13,5 $\mathrm{cm}$, la cola de 10,5-14,5 cm, con un peso de 40-67 g. Habita en bosques siempreverdes, semideciduos y de crecimiento secundario.

Neotoma Representado por la especie N. chrysomelas. Su pelaje es de color café rojizo y la parte ventral blanco grisáceo. Mide 18,5-22 cm de largo, la cola de 15-18 cm, y pesa entre 152-187 g. Se dispone de poca información de esta especie.

Baiomys Género representado por B. musculus. Es un ratón pequeño de tamaño similar al ratón casero (Mus musculus). Se pelaje es de tonalidades de café y gris, con blanco grisáceo en la parte ventral. Mide de 6,5-8 cm, la cola de 4-5,5 cm, con un peso de 8-12 g. Habita en pastizales densos y campos enmalezados.

Scotinomys. Representado por la especie S. teguina. $\mathrm{Al}$ igual que la anterior es un ratón pequeño de tamaño similar al ratón casero (M. musculus). Su coloración es café oscuro y la parte ventral café grisáceo o café anaranjado. Mide de 6,5-8,5 cm, su cola de 5-6 cm, con un peso entre 10-13 $\mathrm{cm}$. Habita en bosques de tierras altas, nubosos, en orillas de bosques.

Reithrodontomys. Este género está representado en Nicaragua por seis especies (Reithrodontomys sumichrasti, $R$. fulcescens, $R$. gracilis, $R$. mexicanus, $R$. brevirostris y $R$. paradoxus). Todas esta especies son de tamaño pequeño, similar al ratón casero (M. musculus), con un largo de cuerpo de 5,5-8 cm, aunque $R$. sumichrasti mide de $6,5-9,5 \mathrm{~cm}$. La cola de estas especies es de unos $8,5-13 \mathrm{~cm}$, y pesan entre 8-15 g. La coloración del pelaje oscila entre café oscuro, café rojizo y café anaranjado, y la parte ventral blanco grisáceo, café blancuzco y blanco amarillento, según la especie. Los hábitats en que se les encuentra son: para $R$. sumichrasti orillas de bosques, matorrales y pastizales y bosques de coníferas, $R$. fulvescens bosques deciduos y áreas con pastizales;
$R$. gracilis bosques maduros, bosques de crecimiento secundario y claros de bosque; $R$. mexicanus bosques maduros, en claros de bosques y áreas cultivadas; $R$. brevirostris bosques siempreverdes y en cafetales con sombra y $R$. paradoxus en bosques deciduos.

Peromyscus Este género está representado en Nicaragua por tres especies (P. mexicanus, $P$. gymnotis y P. stirtoni).

P. mexicanus es de color café oscuro o café grisáceo y la parte ventral blanca. Su cuerpo mide entre $11-13,5 \mathrm{~cm}$, la cola de 10,5-14 cm, y pesan entre 25-50 g. Habita en bosques semideciduos y de crecimiento secundario.

P. gymnotis es de color café grisáceo o negruzco y la parte ventral blanco grisáceo. El cuerpo mide 10-12 cm, la cola 9,5-12 cm y pesa de 29-42 g. Habita en bosques siempreverdes, deciduos y de crecimiento secundario, también se le encuentra en cafetales arbolados.

P. stirtoni es de color café grisáceo y la parte ventral blanca. El cuerpo mide $8,5-11 \mathrm{~cm}$, la cola de $8,5-10,5 \mathrm{~cm}$ y pesa de 24-36 g. Habita en zonas secas, en bosques deciduos y matorrales.

Ratas y ratones exóticos. Las ratas exóticas que se encuentran en Nicaragua pertenecen a la familia Muridae, la cual tiene distribución mundial, y se ha dispersado aprovechando principalmente el transporte de mercancías comerciales (Coto, 2007). En el continente americano está representada por tres especies ampliamente conocidas en las zonas urbanas, la rata negra o de los tejados (Rattus rattus), la rata gris o de caño ( $R$. norvegicus) y el ratón casero o bodeguero (Mus musculus). Estas tres especies son consideradas como las plagas más serias en ambientes urbanos (Battersby et al., 2008).

La rata negra ( $R$. rattus) como su nombre lo indica es de color oscuro con tonalidades de grises hasta negra, aunque hay poblaciones con tonalidades cafesuzcas. Su cuerpo mide entre $16-20 \mathrm{~cm}$, y la cola entre $16,5-24,5 \mathrm{~cm}$. proporciones que se utilizan como referencia para la identificación de las especies de ratas de las zonas urbanas, en este caso con largo de cola que supera al largo del cuerpo (Greaves, 1984). Su nombre común de rata de los tejados hace referencia a su capacidad para subir hasta los techos de edificios. Se encuentra principalmente en casas edificios, bodegas de alimentos de zonas urbanas, y en zonas rurales además de esos sitios se les puede encontrar en área de producción agropecuaria. Es una de las plagas vertebradas más importantes a nivel urbano, cuyos daños se relacionan con el consumo y contaminación de alimentos, daños en las infraestructuras y transmisión de enfermedades (Coto, 2007; Monge, 2009).

La rata gris o de caño ( $R$. norvegicus) tiene un tamaño superior a la rata negra y de igual manera su nombre sugiere su coloración grisácea o cafesuzca grisácea. De igual manera su nombre común de rata de caño obedece a que es común encontrarle en esos sitios, ya que aprovecha los desagües para desplazarse e ingresar a las edificaciones. Su cuerpo 
mide entre $18,5-24 \mathrm{~cm}$ y la cola $12,5-15 \mathrm{~cm}$, lo que de igual manera que se indicó con la rata negra, las proporciones de tamaño del cuerpo y la cola sirven como criterio para la identificación de la especie a nivel urbano, en este caso el largo de la cola es inferior al tamaño del cuerpo (Greaves, 1984). Esta especie también es considerada una de las plagas más importantes a nivel urbano, por el consumo y contaminación de alimentos, daños en las infraestructuras y transmisión de enfermedades. El ratón casero (Mus musculus) es una especie muy conocida, ya que, con frecuencia se le puede encontrar en edificaciones, dada su facilidad para ingresar aprovechando su pequeño tamaño. Su coloración en café grisácea. $\mathrm{Su}$ cuerpo mide $6,5-9 \mathrm{~cm}$, su cola es de tamaño similar al cuerpo y pesa entre 7-15 g. Habita en casas, edificios, bodegas de alimentos y diferentes infraestructuras en ambientes urbanos, aunque también se le puede encontrar en cultivos agrícolas e instalaciones agropecuarias. Los daños referidos a las dos especies de ratas exóticas también se le atribuyen al ratón casero.

Puercoespín. El puercoespín (Sphiggurus mexicanus, anteriormente clasificada como Coendou mexicanus) es un roedor reconocido por la modificación de su pelo a espinas que puede utilizar para defenderse. Es de color negro con las puntas de las espinas amarillo, y la cabeza es color amarillento. El largo de su cuerpo mide $32-46 \mathrm{~cm}$, y su cola prensil de 20$36 \mathrm{~cm}$. Pesa 1,4-2,6 kg. Habita en todo el territorio de Nicaragua, en diferentes tipos de bosques, aunque es poco común en bosques siempreverdes de las tierras bajas de Atlántico.

Guatusa. La guatusa (Dasyprocta punctata) es un roedor diurno, de color café anaranjado. Su cuerpo mide entre 45-57 $\mathrm{cm}$. y prácticamente no tiene cola. Pesa entre 3-4 kg. Habita en bosques deciduos, siempreverdes, de crecimiento secundario y plantaciones. Es más común en áreas protegidas, ya que en otros áreas en fuertemente cazado.

Tepezcuintle. El tepezcuinte (Cuniculus paca) es el roedor nativo más grande de Nicaragua, es de color café rojizo y tiene líneas de manchas blancas en su dorso, la parte ventral es blanca. Mide 50-77,5 cm, no tiene cola y pesa de 5-12 kg. Habita en bosques siempreverdes, deciduos, de crecimiento secundario y jardines. Es común que se encuentre cerca de cuerpos de agua. Es fuertemente cazado por lo que en algunas áreas es escaso o ausente.

Ratas espinosas. En Nicaragua existen dos especies de las llamadas ratas espinosas: Proechimys semispinosus y $\mathrm{Ha}$ plomys gymnurus.

$P$. semispinosus es una rata grande de tamaño superior a la rata de caño. Es de color café rojizo y la parte ventral blanco. Su cuerpo mide entre $22-28 \mathrm{~cm}$, la cola entre 17,5 $19 \mathrm{~cm}$, y pesa $320-536 \mathrm{~g}$. Habita bosque siempreverdes y de crecimiento secundario de tierras bajas.
H. gymnurus al igual que la anterior, también es una rata grande que supera en tamaño a la rata de caño. Mide $20,5-29,5 \mathrm{~cm}$, la cola $12,5-19,5 \mathrm{~cm}$, y pesa entre $230-680 \mathrm{~g}$. Habita en bosque siempreverdes de tierras bajas.

De la lista de roedores registrados para Nicaragua se puede observar que algunas especies pueden relacionarse en mayor medida con el ser humano, ya sea, por los hábitats que ocupan, así como el tamaño de sus poblaciones. Las relaciones pueden calificarse como positivas o negativas, tanto para las personas como para los roedores, lo cual exige en cada caso de un análisis detallado.

En el caso de las ardillas, existen diferencias en cuando a la relación que pueden establecer con las personas. Por ejemplo, la ardilla más común (S. variegatoides) en algunas situaciones se puede considerar una especie plaga porque afecta los cultivos o árboles maderables, mientras que esa misma especie puede ser trofeo para los que practican la caza, o bien ser un animal vistoso lo cual beneficia la actividad turística y como cualquier otra especie forma parte de la biodiversidad en una determinada zona. Otra especie de ardilla (S. richmondi) tiene la particularidad de ser una ardilla endémica de Nicaragua, lo cual requiere de un cuidado especial, dado el impacto ecológico que representaría su extinción.

En cuanto a la taltuza (O. matagalpae, ahora reclasificada como $H$. cherriei) si bien es cierto no es endémica de Nicaragua, dado que se reporta con una distribución limitada en Nicaragua requiere una atención especial para evitar su extinción local. Sin embargo, dado el estilo de vida de esta especie, en caso de encontrarse en zonas de producción agrícola, al igual que otras especies de taltuzas se comporta como una especie dañina y en algunos casos puede poner en riesgo la rentabilidad de la producción agrícola. Ante estas situaciones extremas, la relación que se establece entre este roedor y el ser humano es muy diferente, y por ende deben de plantearse objetivos de manejo distintos.

Las especies de ratas y ratones principalmente de las familias Heteromyidae y Cricetidae, presentan diferencias importantes, que implican relaciones diferentes con las personas. Algunas de estas especies son pequeños ratones poco conocidos que se les encuentra en poblaciones poco densas en diferentes tipos bosques, por lo que su impacto es poco perceptible. Sin embargo, otras especies como la rata cañera o de campo (S. hirsutus) dado que se le encuentra en áreas de producción agrícola y con densidades poblacionales importantes, llega a constituirse en una verdadera plaga agrícola en ciertas épocas de año y en particular en ciertos años, dados los ciclos de la abundancia poblacional.

En el caso de las ratas y el ratón que se encuentra en las áreas urbanas, la relación se considera muy negativa para los intereses de las personas, no solo por los daños en la infraestructura, el consumo y contaminación de alimentos, sino también por la transmisión de enfermedades a personas y animales domésticos. Dada la adaptación de estas especies exótica a los ambientes creados por el ser humano, los cua- 
les les ofrecen alimento, agua y refugio en calidad y cantidad abundante, da lugar a una situación que no forma permanente.

Las ratas llamadas espinosas (P. semispinosus y $H$. gymnurus) no se reportan con ninguna valoración positiva o negativa en Nicaragua; sin embargo, en otros países son aprovechadas para consumo humano (Méndez, 1993).

Finalmente, los roedores de mayor tamaño, a saber, el puercoespín, la guatusa y el tepezcuinte establecen relaciones diferentes con el ser humano. En el caso del puercoespín, aun cuando no es una amenaza para las personas, si puede serlo para animales domésticos, como los perros que intenten atacarlo. La guatusa es un animal que tiene valor ecológico en los sitios en que se encuentra, principalmente en los bosques, ya sea por la depredación o dispersión de semillas, pero además es un animal que en algunos sitios es cazado para el consumo de su carne. De igual manera, el tepezcuinte es un animal que se encuentra en peligro en algunas áreas, producto de la excesiva caza para el consumo de su carne, considerado ésta como una de las mejores carnes de monte.

Por lo anterior, se evidencia que los roedores de Nicaragua es más que ratas y ratones, y aún dentro de este grupo hay diferencias importantes, con relaciones diversas entre estas especies y el ser humano. Por lo tanto, la hablarse de roedores de Nicaragua no se limita a unas pocas especies consideradas plagas, sino que existe una cierta diversidad no solo en términos de cantidad de especies, sino de relaciones positivas y negativas de diversa índole.

En términos generales, aunque se ha referido para unas pocas especies en particular, queda pendiente la tarea de realizar estudios acerca de la biología y ecología de la mayoría de ellas, y aún para aquellas de las que se dispone de algún nivel de conocimiento, es importante corroborarlo a nivel local, de tal manera que si se tienen que tomar algún tipo de acciones de manejo, se haga acorde a las condiciones locales.

\section{REFERENCIAS BIBLIOGRÁFICAS}

Battersby, S., Hirschhorn, R. B.; Amman, B. R. 2008. Commensal rodents. pp. 387-419. In: Bonnefoy, X.; Kampen, H.; Sweeney, K. (eds.). Public health significance of urban pests. World Health Organization. Copenhagen, Denmark. 569 p.

Best, T.L. Sciurus deppei. Mammalian Species 505:1-5.

Coto, H. 2007. Actualización de biología y control de ratas sinantrópicas. Estudio Editorial Gestalt Group. Buenos Aires, Argentina. $571 \mathrm{p}$.

Fleming, T.H. 1991a. Liomys salvini (Ratón semiespinoso, guardafiesta, Spiny Pocket Mouse), pp. 489-491. In: Janzen, D. (Ed). Historia Natural de Costa Rica. Editorial de la Universidad de Costa Rica. San José, Costa Rica. 822 p.

Fleming, T.H. 1991b. Heteromys desmarestianus (Ratón semiespinoso, Spiny Pocket Mouse), pp. 488-489. In: Janzen, D. (Ed). Historia Natural de Costa Rica. Editorial de la Universidad de Costa Rica. San José, Costa Rica. 822 p.

Greaves, J.H. 1984. La lucha contra los roedores en la agricultura. Estudio FAO: Producción y Protección Vegetal 40. Organización de las Naciones Unidas para la Agricultura y la Alimentación. Roma, Italia. 88 p.

Hall, E.R. 1981. The mammals of North America. Second edition. Wiley. New York. 606 p.

Jones, J.K.; Genoways, H.H. 1975. Sciurus richmondi. Mammalian Species 53:1-2.

Méndez, E. 1993. Los roedores de Panamá. Panamá. 372 p.

Monge, J. 2009. Roedores plaga de América Central. Editorial de la Universidad de Costa Rica. San José, Costa Rica.

Reeder, D.M.; Helgen, M.H.; Wilson, D.E. 2007. Global trends and biases in new mammal species discoveries. Occasional Papers. Museum of Texas Tech University. 269:1-35.

Reid, F. A. 2009. A field guide to the mammals of Central America and Southeast Mexico. Second edition. Orford University Press. New York. 346 p.

Straver, C. 1996. Arachis pintoi como cobertura en el cultivo del café: resultados de investigación y experiencia con productores en Nicaragua. p. 150-170. In: Argel, P.J.; Ramírez, P (Eds.). Experiencias regionales con Arachis pintoi y planes futuros de investigación y promoción de la especie en México, Centroamérica y El Caribe. Centro Internacional de Agricultura Tropical (CIAT). Documento de trabajo $\mathrm{N}^{\circ} 159$. Cali, Colombia. $206 \mathrm{p}$.

Wilson, D.E.; Reeder, D.M. 2005. Mammal species of the World. A taxonomic and geographic reference. The Johns Hopkins University Press. Baltimore. USA. 2142 p.

Sánchez C.V; Martínez M.E. 2000. Museum specimen data predict crop damage by tropical rodents. PANS 97(13):7074-7077.

Spradling, T.A; Demastes, J.W; Hafner, D.J; Milbach, P.L; Cervantes, F.A. 2016. Systematic revision of the pocket gopher genus Orthogeomys. J. Mamm. 97:405-423.

Weksler, M; Percequillo, A.R; Voss, R.S. 2006. Ten New Genera of Oryzomyine Rodents (Cricetidae: Sigmodontinae). American Museum Novitates 3537:1-29

Wingching-Jones, R; Monge-Meza, J; Pérez-Salas, R. 2009. Roedores pequeños en un sistema de producción de ganado lechero. Agronomía Mesoamericana 20(2):127-133. 